\title{
THE STONE-ČECH COMPACTIFICATION OF AN IRREDUCIBLY CONNECTED SPACE
}

\author{
GLENN L. PFEIFER
}

1. Introduction. A connected topological space $X$ is irreducibly connected about a subset $A \subset X$ (written $X$ is an $A$-i-connex) if no proper connected subspace of $X$ contains $A$. A connected space $Y$ is irreducibly closed connected about a subset $B \subset Y$ (written $Y$ is a $B-i$-C-connex) if no proper closed connected subspace of $Y$ contains $B$. The structure of such spaces has been studied by Gehman [1], Wilder [7] and Strebe [3], [4], among others. In this paper we show that the Stone-Cech compactification $\beta X$ of an $i$-connex $X$ is an $i$-connex, and that $\beta Y$, for $Y$ a suitably restricted $i$ - $C$-connex, is an $i$ - $C$-connex.

The author would like to express his appreciation to Professor Edwin Halfar for many conversations concerning this paper.

2. Notation. All spaces are at least $T_{1}$ and completely regular. For $S \subset X, C l S$ is the closure of $S$ in $X$, and for $T \subset \beta X, \mathrm{Cl}_{\beta} T$ is the closure of $T$ in $\beta X$. If $S \subset X$, then $S^{0}=\beta X-\mathrm{Cl}_{\beta}(X-S)$.

\section{3. $i$-connexes.}

THEOREM 1. If $X$ is an $A$-i-connex, then $\beta X$ is an $A \cup(\beta X-X)-i$ connex.

Proof. Since $X$ is connected, each point of $\beta X-X$ is a noncut point of $\beta X$. Thus any set about which $\beta X$ is an $i$-connex must contain $\beta X-X[1$, Theorem 3, p. 545].

If there is a subspace $X^{\prime} \neq \beta X$ with $A \cup(\beta X-X) \subset X^{\prime}$, then for any $x \in \beta X-X^{\prime}, x \in X-A$ so $x$ is a cut point of $X$. However, any cut point of $X$ is a cut point of $\beta X$ because an open subset $U$ of $\beta X$ is connected if and only if $U \cap X$ is connected [2, Lemma 1.4, p. 575]. Thus for $x \in \beta X-X^{\prime}, \beta X-\{x\}=P \cup Q$ (sep).

If $X^{\prime}$ is connected, then $X^{\prime} \subset P$, say. Now $Q \cup\{x\}$, being a continuum, has a noncut point $z \neq x[5$, Theorem 1.11, p. 491]. Further $z \in X-A$. It follows that

$$
\beta X-\{z\}=(P \cup\{x\}) \cup[(Q \cup\{x\})-\{z\}]
$$

is connected; hence that $X-\{z\}$ is a connected proper subspace of $X$ containing $A$, a contradiction.

Presented to the Society, January 24, 1968; received by the editors July 20, 1967. 
To see that no converse is possible, let $X$ be the nonnegative reals with the usual topology and $A=\{0\}$.

\section{4. $i$-C-connexes.}

Theorem 2. If the $A-i-C$-connex $X$ is normal and is semilocally connected at each $x \in X-A$, then $\beta X$ is an $A-i-C$-connex.

Proof. If there is a proper closed connected subspace $K$ of $\beta X$ with $A \subset K$, then for $x \in X \cap(\beta X-K)$ there is an $X$-open neighborhood $V$ of $x$ with $\mathrm{Cl}_{\beta} V \cap K=\varnothing$. Since $X$ is semilocally connected at $x$, there is an $X$-open set $U$ with $x \in U \subset V$ such that $X-U$ has finitely many components $C_{1}, \cdots, C_{n}$. Further, $n \geqq 2$ and $A \cap C_{i} \neq \varnothing$ for at least two indices $i$.

Now $X$ is normal and therefore the closures in $\beta X$ of $C_{1}, \cdots, C_{n}$ are mutually disjoint closed connected sets [6, Theorem 1, p. 97], i.e., they are the components of $\mathrm{Cl}_{\beta}(X-U)$. It follows that $A \subset K \subset \mathrm{Cl}_{\beta} C_{j}$ for some index $j$, a contradiction.

Theorem 3. If the $A-i-C$-connex $X$ is normal and locally connected, then $\beta X$ is an $A-i-C$-connex.

Proor. If there is a proper closed connected subspace $K$ of $\beta X$ with $A \subset K$, then there is a connected $X$-open set $U$ with $\mathrm{Cl}_{\beta} U \cap K=\varnothing$. $X-U$ is not connected, so from $X-U=\mathrm{Cl}(X-\mathrm{Cl} U)$ it follows that $X-\mathrm{Cl} U$ is not connected.

If $C$ is a component of $X-\mathrm{Cl} U$, then $C$ is open in $X$ and $X-C$ is closed and connected. Therefore $C \cap A \neq \varnothing$ for each component $C$ of $X-\mathrm{Cl} U$. Thus $X-\mathrm{Cl} U=P \cup Q($ sep) with $P \cap A \neq \varnothing, Q \cap A \neq \varnothing$.

Now $K \subset \beta X-\mathrm{Cl}_{\beta} U=(X-\mathrm{Cl} U)^{0}=(P \cup Q)^{0}$, and by [6, Lemma 2, p. 98] $(P \cup Q)^{0}=P^{0} \cup Q^{0}$ and $(P \cap Q)^{0}=P^{0} \cap Q^{0}$. Since $K$ is connected, $K \subset P^{0}$, say; however, $A \cap Q^{0} \supset A \cap Q \neq \varnothing$ and this is a contradiction.

\section{BIBLIOGRAPHY}

1. H. M. Gehman, Concerning irreducibly connected sets and irreducible continua, Proc. Nat. Acad. Sci. U.S.A. 12 (1926), 544-547.

2. M. Henriksen and J. R. Isbell, Local connectedness in the Stone-Cech compactification, Illinois J. Math. 1 (1957), 574-582.

3. D. D. Strebe, Irreducibly connected spaces, Duke Math. J. 20 (1953), 551-561.

4. - Irreducible closed connexes, Duke Math. J. 22 (1955), 365-372.

5. A. D. Wallace, Monotone transformations, Duke Math. J. 9 (1942), 487-506.

6. - Extensional invariance, Trans. Amer. Math. Soc. 70 (1951), 97-102.

7. R. L. Wilder, Concerning irreducibly connected sets and irreducible regular connexes, Amer. J. Math. 56 (1934), 547-557.

UNIVERSITY OF ARIZONA 